\title{
Padrões de floração e frutificação de árvores da Amazônia Maranhense
}

\author{
Francisca Helena MUNIZ1
}

\begin{abstract}
RESUMO
Estudos fenológicos em nível de comunidades podem facilitar a compreensão do comportamento das espécies diante de alterações nos ecossistemas, além de refletir a distribuição anual de tipos específicos de recursos. Este trabalho buscou definir os padrões gerais e a sazonalidade de floração e frutificação de uma comunidade em duas áreas de floresta na Amazônia Maranhense, uma não perturbada e outra submetida a corte seletivo. A vegetação corresponde às matas de cipós das florestas amazônicas, alternando matas densas e abertas, de alta biomassa. Valores médios anuais de temperatura variam entre $24,5^{\circ} \mathrm{C}$ e $26,0^{\circ} \mathrm{C}$, e entre 1400 $\mathrm{mm}$ e $1800 \mathrm{~mm}$ de precipitação, com um período seco de 5 a 6 meses, de junho a novembro. Foram analisadas a floração e a frutificação de 89 espécies arbóreas, de agosto de 1994 a junho de 1996. As espécies foram agrupadas em: árvores do sub-dossel, árvores do estrato superior e árvores que ocorrem em ambos os estratos. Foi feita comparação entre grupos (estratos, tipos de floresta e mecanismos de dispersão) e possíveis correlaçōes com a precipitação foram investigadas. Quinze espécies estudadas foram exclusivas do estrato inferior e 63 do estrato superior da floresta; 17 espécies foram registradas apenas na mata nativa e 37 apenas na mata manejada. A maioria das espécies é zoocórica (62,9\%). A floração e a frutificação ocorreram durante todo o ano, com pico de floração de outubro a dezembro e picos de frutificação de março a julho e de outubro a dezembro. Os resultados obtidos demonstram uma grande sincronia na floração e frutificação dos indivíduos, e confirmam a relação entre esses processos e a variação na precipitação ao longo do ano, e que plantas de ambientes diferenciados exibem comportamentos fenológicos diferentes. Os padrōes observados foram semelhantes entre as áreas e a outros estudos na Amazônia.
\end{abstract}

PalaVraS-CHAVE: Fenologia; Padrões de floração; Padrões de frutificação; Floresta Amazônica Maranhense.

\section{Flowering and Fruiting Patterns of the Maranhense Amazon Rainforest Trees}

\section{ABSTRACT}

Community level phonological studies can facilitate the understanding of species behavior as a result of ecosystem changes, further reflecting on the annual allotment of specific resources. The aim of the present study was to define the general patterns, flowering and fruiting seasonality from a community in two forest areas of the Maranhense Amazon Rainforest: a non-disturbed area and another submitted to selective logging. The vegetation is composed of Amazon forest lianas alternating between dense and open high biomass forest. Average annual temperature varies between $24.5^{\circ} \mathrm{C}$ and $26.0^{\circ} \mathrm{C}$, with precipitation ranging from $1400 \mathrm{~mm}$ to $1800 \mathrm{~mm}$, and a dry season between June and November. Flowering and fruiting of 89 species were analyzed from August 1994 to June 1996. The species were grouped as follows: sub-dossal, upper strata, and trees occurring in both strata. Comparison was made between groups (strata, types of forest and mechanisms for dispersal) and possible correlations with rainfall were investigated. Fifteen studied species were solely from the lower strata, and 63 from the upper forest strata; 17 species were recorded only in native forest and 37 in managed forest. Most species $(62.9 \%)$ is zoochorous. Flowering and fruiting take place throughout the year with flowering peak from October to December and fruiting peaks from March to July and from October to December. The results showed a great synchrony in flowering and fruiting of individuals, and confirm the relationship between these cases and the variation in rainfall throughout the year, and that plants of different environments exhibit phonological behavior different. The observed flowering and fruiting patterns were similar between the areas and comparable to other studies in the Amazon Rainforest.

KEY-WORDS: Phenology; Flowering patterns; fruiting patterns; Maranhense Amazon Rainforest 


\section{INTRODUÇÃO}

A organização biológica dos sistemas tropicais, e sua evolução, pode ser melhor compreendida através da análise fenológica de comunidades. A periodicidade nos padrôes reprodutivos e vegetativos das plantas reflete a distribuição anual e a disponibilidade de tipos específicos de recursos (Frankie et al., 1974a).

Estudos em florestas tropicais úmidas sugerem que os padrōes fenológicos das árvores são dirigidos por uma variedade de fatores, incluindo características abióticas como precipitação, irradiação e temperatura (Ashton et al., 1988; Newbery et al., 1998; Opler et al., 1976; Van Schaik, 1986; Van Schaik et al., 1993; Sun et al., 1996; Tutin \& Fernandez, 1993; Chapman et al., 1999; Chapman et al., 2005; Zimmerman et al., 2007); modo de dispersão de sementes (Charles-Dominique et al., 1981; Smythe, 1970; Snow, 1965; Wheelwright, 1985); atividade de polinizadores e dispersores de sementes (Frankie et al., 1974a; Rathke \& Lacey, 1985; Snow, 1965); variação nas condiçōes de germinação (Frankie et al., 1974b; Janzen, 1967); posição no dossel (Newstrom et al., 1994); e abundância relativa das árvores (Van Schaik et al., 1993).

$\mathrm{Na}$ Amazônia, informaçōes sobre a fenologia das espécies são ainda escassas e fragmentadas, e os dados existentes dizem respeito, principalmente, àquelas espécies de interesse ou potencial econômico (Araújo, 1970; Macêdo, 1977; Alencar et al., 1979; Carvalho, 1980; Gribel et al., 1999; Pinto et al., 2005).

A característica mais significante de florestas tropicais úmidas é o calor e a umidade contínuos, o que favorece o crescimento ao longo do ano (Richards, 1952) e resulta em uma diversidade de estratégias e padrôes fenológicos maior que em outros ecossistemas (Sarmiento \& Monastério, 1983; Newstrom et al., 1994). Os ciclos fenológicos de plantas tropicais são complexos, apresentando padrōes irregulares de difícil reconhecimento, principalmente em estudos de curto prazo (Newstrom et al., 1994; Bencke \& Morellato, 2002).

Pouco se conhece sobre as épocas de floração e frutificação das espécies arbóreas na floresta amazônica maranhense de terra firme, assim este trabalho buscou definir os padrōes gerais de uma comunidade florestal ao longo do ano, relacionando com as estaçōes do ano, os estratos em que as espécies ocorrem e suas síndromes de dispersão, contrastando uma área de floresta nativa e outra de floresta manejada no município de Buriticupu, admitindo-se como hipótese que os padrōes observados podem ser influenciados pelo manejo dessas florestas.

\section{MATERIAL E MÉTODOS}

\section{LOCAL DE ESTUDO}

Este trabalho foi realizado no município de Buriticupu (4º' 16 S e $\left.46^{\circ} 10^{\prime} \mathrm{W}\right)$, no estado do Maranhão, sudoeste da Amazônia. O solo é predominantemente Latossolo Vermelho Amarelo A moderado, textura argilosa, com boa drenagem interna, em relevo plano a suavemente ondulado, com declividade até $25^{\circ}$ e altitude média de $350 \mathrm{~m}$ (Ramos et al., 1991).

O clima, de acordo com o Sistema de Classificação de Köppen, é do tipo "Aw", úmido com moderado déficit de água, com temperatura média anual entre 24,5 e $26,0{ }^{\circ} \mathrm{C}$ e precipitação média anual entre 1400 e $1800 \mathrm{~mm}$. Há um período seco de cinco a seis meses, de junho a novembro, quando o déficit hídrico atinge 150-300 mm (Golfari, 1980; Jesus et al., 1986), situando-se o período mais seco entre julho e outubro, com a maior concentração de chuvas em fevereiro e março (Brasil, 1986) (Fig. 1).

A vegetação corresponde às matas de cipó das florestas amazônicas. É uma formação com alternância de matas densas e abertas, de biomassa mediana, apresentando uma transição gradual desde o tipo florestal mais úmido até o tipo semidecíduo, à medida que se desloca para o sul (Golfari, 1980).

$\mathrm{O}$ trabalho foi realizado em uma área de vegetação nativa e uma submetida à corte seletivo, na qual foi instalado um ensaio de manejo florestal, com a eliminação de cipós e retirada de árvores com DAP igual ou superior a $45 \mathrm{~cm}$ (Jesus et al., 1986). Não foi feita nenhuma intervenção posterior, e a área, cerca de 11 anos depois, apresentava-se em avançado estágio de recuperação.

Estudo realizado por Muniz (1998) mostrou que ambas as áreas apresentam alta biomassa e grande diversidade de espécies. A floresta nativa apresentou área basal das árvores com DAP (Diâmetro a Altura do Peito) igual ou superior a $9,5 \mathrm{~cm}$ de $41,896 \mathrm{~m}^{2} \cdot \mathrm{ha}^{-1}$, índice de diversidade de Shannon

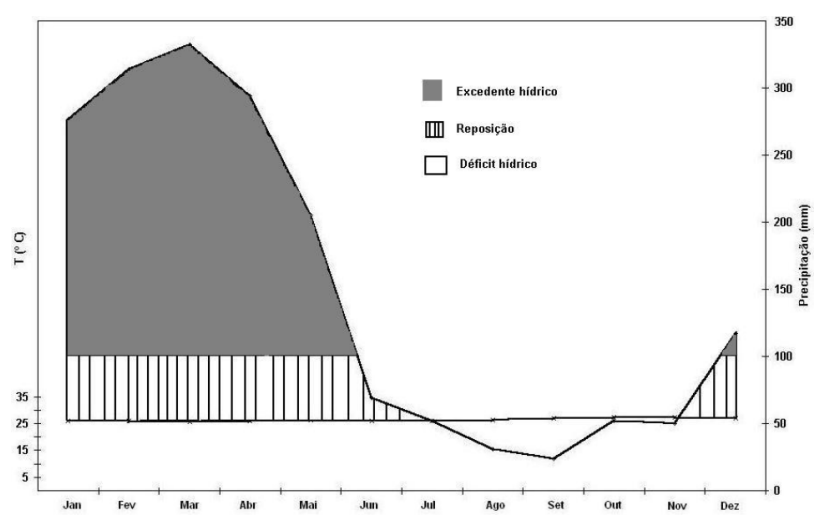

Figura 1 - Diagrama climático, município de Buriticupu, Maranhão, Sudoeste da Amazônia (de acordo com Walter, 1986). 
\& Weaver $\left(\mathrm{H}^{\prime}\right)$ de 3,350 nats.ind ${ }^{-1}$, densidade de 570 ind. $\mathrm{ha}^{-1} \mathrm{e}$ altura média das árvores em torno de 17,2 m. Na floresta manejada, a área basal das árvores com DAP $\geq 9,5$ $\mathrm{cm}$ foi $24,694 \mathrm{~m}^{2}$.ha ${ }^{-1}, \mathrm{H}^{\prime}$ de 3,67 nats.ind ${ }^{-1}$, densidade de 639 ind.ha ${ }^{-1}$ e altura média das árvores em torno de $15,8 \mathrm{~m}$ (Muniz, 1998).

\section{COLETA DE DADOS}

Os dados foram coletados mensalmente entre agosto de 1994 e junho de 1996. Foram anotados os períodos de floração (botôes florais e flores) e de frutificação (frutos novos e maduros) de cada indivíduo arbóreo marcado em um levantamento florístico e fitossociológico realizado na área (Muniz, 1998).

Foi considerado o período total de ocorrência (ou atividade) da fenofase para cada espécie, e o número de espécies e indivíduos que apresentaram a fenofase foi usado como um indicador da intensidade da respectiva fase fenológica.

As espécies foram agrupadas em: árvores e arvoretas do sub-dossel (aquelas com DAP $<9,5 \mathrm{~cm}$ ), árvores do dossel e estrato superior (aquelas com DAP $\geq 9,5 \mathrm{~cm}$ ), e árvores e arvoretas que ocorrem em ambos os estratos.

As espécies foram separadas de acordo com seus mecanismos de dispersão, em zoocóricas, anemocóricas e autocóricas, considerando-se a morfologia dos frutos ou a literatura (Pijl, 1982; Roosmalen, 1985; Fischer \& Chapman, 1993) quando disponível.

A normalidade dos dados foi testada com os testes Kolmogorov-Smirnov e Lilliefor's e foi refutada. Como também não foi possível atingir normalidade por transformação de dados, foram utilizados testes não-paramétricos. A comparação entre grupos (estratos, tipos de floresta e mecanismos de dispersão) foi feita com o teste ' $U$ ' de Mann-Whitney e possíveis correlações com a precipitação foram investigadas utilizando-se Spearman. O teste Mann-Whitney (U) foi utilizado para checar uma possível diferença sazonal na floração e frutificação, classificando-se a precipitação em estação úmida (precipitação $\geq 100 \mathrm{~mm}$ por mês) e estação seca (precipitação < $100 \mathrm{~mm}$ por mês) (Zar, 1996).

\section{RESULTADOS E DISCUSSÃO}

Foram registradas 89 espécies, pertencentes a 62 gêneros e 29 famílias, que floresceram ou frutificaram durante o período de estudo. Destas, 25 foram observadas apenas com flores, 21 apenas com frutos e 43 floresceram e frutificaram. Como as observações foram conduzidas somente uma vez por mês, períodos efêmeros de floração e frutificação podem ter sido omitidos. Durante todo o ano ocorreram espécies em flor ou fruto (Tabela 1).
Das espécies que floresceram e/ou frutificaram no período, 15 foram exclusivas do sub-bosque, 63 do dossel e 11 de ambos os estratos. Dezessete espécies foram observadas apenas na mata nativa, 37 na mata manejada e 35 em ambas. O número de indivíduos por espécie variou de 1 a 89 , devido às variações em abundância das espécies na área.

No nível da comunidade, observou-se um padrão contínuo de floração com picos sazonais, o que parece ser típico de florestas tropicais úmidas (Richards, 1952; Whitmore, 1984; Longman \& Jenik, 1987; Newstrom et al., 1994; Chapman et al., 1999; Haugaasen e Peres, 2005). Existiu um período grande de floração de julho a abril, com um pico em novembro/dezembro, início de estação chuvosa (Fig. 2A). Frankie et al. (1974b) observaram que mais espécies floresceram no pico da estação seca e início da estação chuvosa, enquanto menos espécies floresceram no final da estação úmida na Costa Rica. Em florestas sujeitas a regime sazonal de chuvas, muitas espécies do dossel e do sub-dossel produzem flores no início da estação seca (Frankie et al., 1974a; Opler et al., 1980). O período de máxima intensidade de floração (Fig. 2B) coincidiu com o máximo da estação chuvosa, e está possivelmente relacionado com a estação de dispersão dos frutos, com a disponibilidade de agentes dispersores, e com as condições mais favoráveis para a germinação das sementes (Foster, 1985). Isto resulta em uma grande sincronia entre os indivíduos da comunidade.

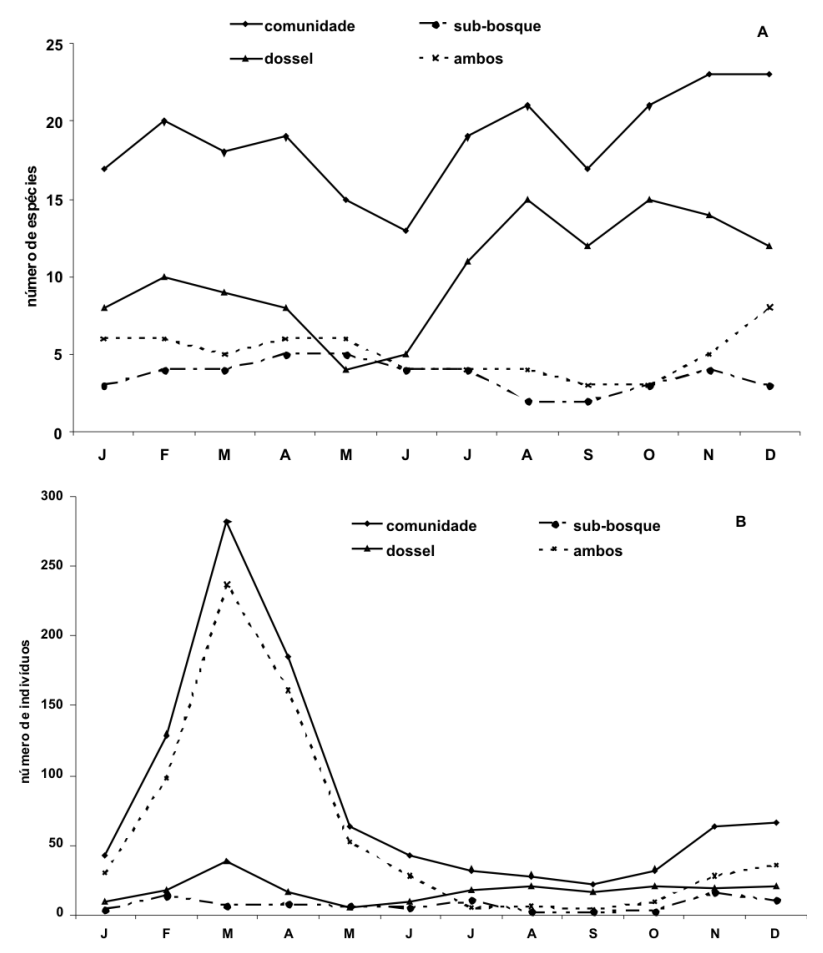

Figura 2 - Número de espécies arbóreas (A) e de indivíduos (B) em floração da comunidade e por estrato (dossel, sub-bosque, e ambos (dossel e subbosque)), Buriticupu, Maranhão, Sudoeste da Amazônia. 
Tabela 1 - Relação de espécies com suas respectivas fases de floração e frutificação, mecanismo de dispersão e posição que ocupa (sub-bosque; dossel; ambos os estratos) na Amazônia Maranhense, Buriticupu - MA.

\begin{tabular}{|c|c|c|c|c|c|c|c|}
\hline Família & Espécie & Nome vulgar & Floração & Frutificação & $\begin{array}{l}\text { Mecanismo de } \\
\text { dispersão }\end{array}$ & $\begin{array}{l}\text { Posição no } \\
\text { dossel }\end{array}$ & Ambiente \\
\hline Achariaceae & Lindackeria latifolia Benth. & & Jan./Fev. & & Zoocoria & dossel & mata nativa \\
\hline \multirow[t]{2}{*}{ Anacardiaceae } & Astronium gracile Engl. & Muiracatiara & Set. & & Anemocoria & dossel & mata manejada \\
\hline & Spondias lutea L. & Cajazinho & & Mar./Maio & Zoocoria & dossel & mata nativa \\
\hline \multirow[t]{5}{*}{ Annonaceae } & Duguetia duckei R.E. Fr. & & Jul./Dez. & & Zoocoria & dossel & mata manejada \\
\hline & Duguetia echinophora R.E. Fr. & Atameijú & Ago./Jan. & Jan./Dez. & Zoocoria & ambos & $\begin{array}{l}\text { mata nativa e } \\
\text { manejada }\end{array}$ \\
\hline & Guatteria poeppigiana Mart. & Envira preta & & Abr./Jul. & Zoocoria & dossel & mata manejada \\
\hline & Oxandra reticulata Maas & Condurú & Jul./Nov. & Out./Jan. & Zoocoria & dossel & $\begin{array}{l}\text { mata nativa e } \\
\text { manejada }\end{array}$ \\
\hline & Rollinia exsucca (DC. ex Dunal) A. DC. & Mutamba preta & & Abr./Jul. & Zoocoria & dossel & mata manejada \\
\hline Apocynaceae & $\begin{array}{l}\text { Tabernaemontana flavicans Willd. ex } \\
\text { Roem. \& Schultz. }\end{array}$ & & Ago./Out. & & Anemocoria & sub-bosque & mata nativa \\
\hline \multirow[t]{2}{*}{ Bignoniaceae } & $\begin{array}{l}\text { Tabebuia impetiginosa (Mart. ex DC.) } \\
\text { Standl. }\end{array}$ & Ipê roxo & Jul. & & Anemocoria & dossel & mata nativa \\
\hline & $\begin{array}{l}\text { Tabebuia serratifolia (Vahl.) G. } \\
\text { Nicholson }\end{array}$ & Ipê amarelo & Ago./Out. & Set./Out. & Anemocoria & dossel & $\begin{array}{l}\text { mata nativa e } \\
\text { manejada }\end{array}$ \\
\hline Boraginaceae & Cordia scabrifolia A. DC. & Freijó & & Ago./Dez. & Anemocoria & dossel & mata manejada \\
\hline \multirow[t]{2}{*}{ Brassicaceae } & Capparis amazonica H.H. Iltis & Feijão brabo & Out./Dez. & Jan./Abr. & Zoocoria & sub-bosque & $\begin{array}{l}\text { mata nativa e } \\
\text { manejada }\end{array}$ \\
\hline & Capparis elegans Mart. & & & Out./Nov. & Zoocoria & sub-bosque & mata nativa \\
\hline \multirow[t]{2}{*}{ Burseraceae } & $\begin{array}{l}\text { Protium heptaphyllum (Aubl.) } \\
\text { Marchand }\end{array}$ & $\begin{array}{l}\text { Amesclão } \\
\text { branco }\end{array}$ & & Out./Nov. & Zoocoria & dossel & mata manejada \\
\hline & Protium tenuifolium (Engl.) Engl. & Amesclão roxo & Jul./Set. & Jul./Fev. & Zoocoria & dossel & $\begin{array}{l}\text { mata nativa e } \\
\text { manejada }\end{array}$ \\
\hline Caricaceae & Jacaratia spinosa (Aubl.) A. DC. & Mamuí & & Dez./Mar. & Zoocoria & dossel & mata manejada \\
\hline Euphorbiaceae & Croton cajucara Benth. & Imbaca & Nov./Jun. & Maio/Dez. & Autocoria & dossel & $\begin{array}{l}\text { mata nativa e } \\
\text { manejada }\end{array}$ \\
\hline \multirow[t]{7}{*}{$\begin{array}{l}\text { Fabaceae-Cae- } \\
\text { salpinioideae }\end{array}$} & Cenostigma gardnerianum Tul. & Caneleiro & Jan./Dez. & Fev./Dez. & Anemocoria & dossel & $\begin{array}{l}\text { mata nativa e } \\
\text { manejada }\end{array}$ \\
\hline & $\begin{array}{l}\text { Chamaecrista xinguensis (Ducke) H.S. } \\
\text { Irwin \& Barneby }\end{array}$ & Pituruna & Jan./Dez. & Out./Dez. & Anemocoria & ambos & $\begin{array}{l}\text { mata nativa e } \\
\text { manejada }\end{array}$ \\
\hline & Copaifera reticulata Ducke & Copaíba & & Out./Mar. & Zoocoria & dossel & $\begin{array}{l}\text { mata nativa e } \\
\text { manejada }\end{array}$ \\
\hline & Dialium guianense (Aubl.) Sandwith & Jutaí-pororoca & & Jan. & Zoocoria & dossel & mata manejada \\
\hline & Hymenaea courbaril L. & Jatobá de fava & Out./Dez. & Dez./Ago. & Zoocoria & dossel & $\begin{array}{l}\text { mata nativa e } \\
\text { manejada }\end{array}$ \\
\hline & Hymenaea parvifolia Huber & Jatobá curuba & Ago./Dez. & Jan./Dez. & Zoocoria & dossel & $\begin{array}{l}\text { mata nativa e } \\
\text { manejada }\end{array}$ \\
\hline & Macrolobium multijugum (DC.) Benth. & & & Jun./Out. & Anemocoria & dossel & mata nativa \\
\hline \multirow[t]{2}{*}{$\begin{array}{l}\text { Fabaceae- } \\
\text { Cercideae }\end{array}$} & Bauhinia angulata L. & Capa-bode & Fev./Mar. & Mar./Jul. & Zoocoria & dossel & $\begin{array}{l}\text { mata nativa e } \\
\text { manejada }\end{array}$ \\
\hline & Bauhinia macrostachya Benth. & Mororó & Abr./Jun. & Abr./Jul. & Zoocoria & ambos & $\begin{array}{l}\text { mata nativa e } \\
\text { manejada }\end{array}$ \\
\hline \multirow[t]{5}{*}{$\begin{array}{l}\text { Fabaceae- } \\
\text { Faboideae }\end{array}$} & Dipteryx odorata (Aubl.) Willd. & Cumaru & Out./Maio & & Anemocoria & dossel & $\begin{array}{l}\text { mata nativa e } \\
\text { manejada }\end{array}$ \\
\hline & Platypodium elegans Vogel & Três quinas & Nov./Jan. & Mar./Dez. & Anemocoria & dossel & mata manejada \\
\hline & Swartzia flaemingii Raddi & $\begin{array}{l}\text { Jacarandá de } \\
\text { veado }\end{array}$ & Mar./Maio & Mar./Nov. & Anemocoria & dossel & $\begin{array}{l}\text { mata nativa e } \\
\text { manejada }\end{array}$ \\
\hline & Swartzia laurifolia Benth. & & & Dez. & Anemocoria & dossel & mata manejada \\
\hline & Zollernia paraensis Huber & pau santo & Ago./Out. & Nov./Dez. & Anemocoria & dossel & $\begin{array}{l}\text { mata nativa e } \\
\text { manejada }\end{array}$ \\
\hline
\end{tabular}


Tabela 1 - Continuação

\begin{tabular}{|c|c|c|c|c|c|c|c|}
\hline Família & Espécie & Nome vulgar & Floração & Frutificação & $\begin{array}{l}\text { Mecanismo de } \\
\text { dispersão }\end{array}$ & $\begin{array}{l}\text { Posição no } \\
\text { dossel }\end{array}$ & Ambiente \\
\hline \multirow[t]{2}{*}{$\begin{array}{l}\text { Fabaceae-Mimo- } \\
\text { soideae }\end{array}$} & Inga falcistipula Ducke & & Jun. & & Zoocoria & dossel & mata manejada \\
\hline & Parkia sp. & Faveira & & Maio/Jul. & Zoocoria & dossel & mata nativa \\
\hline \multirow[t]{2}{*}{ Lamiaceae } & Aegiphila amazonica Moldenke & & Jun./Set. & & Zoocoria & sub-bosque & mata manejada \\
\hline & Vitex schomburgkiana Schauer & & Dez. & & Zoocoria & dossel & mata nativa \\
\hline \multirow[t]{3}{*}{ Lecythidaceae } & Gustavia augusta L. & Juruparana & Out./Dez. & Out./Dez. & Zoocoria & sub-bosque & mata nativa \\
\hline & $\begin{array}{l}\text { Holopyxidium latifolium (Ducke) R. } \\
\text { Knuth }\end{array}$ & Inhaúba & Jul./Out. & Out. & Zoocoria & dossel & $\begin{array}{l}\text { mata nativa e } \\
\text { manejada }\end{array}$ \\
\hline & Lecythis lurida (Miers) S.A. Mori & Jarana & Ago./Nov. & Dez./Fev. & Zoocoria & dossel & mata manejada \\
\hline \multirow[t]{3}{*}{ Malvaceae } & Apeiba albiflora Ducke & $\begin{array}{l}\text { Pente de } \\
\text { macaco }\end{array}$ & Dez. & & Zoocoria & dossel & mata nativa \\
\hline & Ceiba sp. & $\begin{array}{l}\text { Espinho de } \\
\text { roseta }\end{array}$ & & Dez. & Anemocoria & dossel & mata manejada \\
\hline & Erioteca sp. & Algodoeiro & & Jul./Nov. & Anemocoria & dossel & $\begin{array}{l}\text { mata nativa e } \\
\text { manejada }\end{array}$ \\
\hline \multirow[t]{2}{*}{ Meliaceae } & Trichilia quadrijuga Kunth & & & Out. & Zoocoria & dossel & mata nativa \\
\hline & Trichilia sp. & Cedrinho & Ago./Fev. & & Zoocoria & dossel & $\begin{array}{l}\text { mata nativa e } \\
\text { manejada }\end{array}$ \\
\hline \multirow[t]{4}{*}{ Myrtaceae } & Eugenia protracta Steud. & & Ago./Nov. & Ago./Fev. & Zoocoria & dossel & mata manejada \\
\hline & Eugenia sp.1 & & Jul./Fev. & Abr./Dez. & Zoocoria & dossel & $\begin{array}{l}\text { mata nativa e } \\
\text { manejada }\end{array}$ \\
\hline & Eugenia sp.2 & & Jan./Mar. & Mar./Maio & Zoocoria & dossel & $\begin{array}{l}\text { mata nativa e } \\
\text { manejada }\end{array}$ \\
\hline & Myrcia eximia DC. & & Out. & & Zoocoria & dossel & mata nativa \\
\hline Nyctaginaceae & Guapira hirsuta (Choisy) Lundell & Maria mole & Dez./Fev. & Dez./Mar. & Zoocoria & ambos & $\begin{array}{l}\text { mata nativa e } \\
\text { manejada }\end{array}$ \\
\hline Picrodendraceae & Piranhea trifoliata Baill. & Piranheira & Ago./Set. & Out. & Anemocoria & dossel & mata nativa \\
\hline Phyllanthaceae & Margaritaria nobilis L. f. & Capoeiro & & Dez./Maio & Zoocoria & ambos & mata manejada \\
\hline Rhamnaceae & Zizyphus itacaiunensis Froes & Maria preta & Abr. & Jun./Set. & Zoocoria & dossel & $\begin{array}{l}\text { mata nativa e } \\
\text { manejada }\end{array}$ \\
\hline \multirow[t]{3}{*}{ Rubiaceae } & Alseis floribunda Schott & $\begin{array}{l}\text { Escorrega- } \\
\text { macaco }\end{array}$ & Out./Dez. & Jul. & Anemocoria & dossel & $\begin{array}{l}\text { mata nativa e } \\
\text { manejada }\end{array}$ \\
\hline & Faramea bracteata Benth. & Folha grossa & Jan./Mar. & Fev./Jul. & Zoocoria & dossel & mata manejada \\
\hline & Guettarda angelica Mart. ex Müll. Arg. & Angélica & Nov./Jan. & Mar./Maio & Zoocoria & dossel & mata manejada \\
\hline Rubiaceae & Ixora martinsii Standl. & & Jul. & & Zoocoria & dossel & mata manejada \\
\hline \multirow[t]{9}{*}{ Rutaceae } & Cusparia toxicaria Spruce ex Engl. & Bolotinha & Nov./Jul. & Set./Abr. & Autocoria & sub-bosque & $\begin{array}{l}\text { mata nativa e } \\
\text { manejada }\end{array}$ \\
\hline & Esenbeckia almawillia Kaastra & Amarelinho & Fev./Jul. & Fev./Set. & Autocoria & sub-bosque & $\begin{array}{l}\text { mata nativa e } \\
\text { manejada }\end{array}$ \\
\hline & Galipea trifoliata Aubl. & $\begin{array}{l}\text { Laranjinha } \\
\text { folha fina }\end{array}$ & Dez./Jul. & Jul./Dez. & Autocoria & ambos & mata manejada \\
\hline & Metrodorea flavida K. Krause & $\begin{array}{l}\text { Laranjinha } \\
\text { folha grossa }\end{array}$ & Nov./Ago. & Jul./Dez. & Autocoria & ambos & $\begin{array}{l}\text { mata nativa e } \\
\text { manejada }\end{array}$ \\
\hline & Neoraputia magnifica Emmerich. & $\begin{array}{l}\text { Café brabo flor } \\
\text { rosa }\end{array}$ & Fev./Maio & Mar./Dez. & Autocoria & ambos & $\begin{array}{l}\text { mata nativa e } \\
\text { manejada }\end{array}$ \\
\hline & $\begin{array}{l}\text { Pilocarpus microphyllus Stapf ex } \\
\text { Holmes }\end{array}$ & Jaborandi & Abr./Maio & Maio/Ago. & Zoocoria & sub-bosque & mata manejada \\
\hline & Pilocarpus sp. & Arrudão & & Set. & Zoocoria & sub-bosque & mata manejada \\
\hline & Rauia resinosa Nees \& C. Mart. & $\begin{array}{l}\text { Café brabo flor } \\
\text { branca }\end{array}$ & Dez./Maio & Mar./Dez. & Autocoria & ambos & $\begin{array}{l}\text { mata nativa e } \\
\text { manejada }\end{array}$ \\
\hline & Zanthoxylum rhoifolium Lam. & Limãozinho & Jan./Mar. & Abr./Maio & Autocoria & dossel & mata manejada \\
\hline
\end{tabular}


Tabela 1 - Continuação

\begin{tabular}{|c|c|c|c|c|c|c|c|}
\hline Família & Espécie & Nome vulgar & Floração & Frutificação & $\begin{array}{l}\text { Mecanismo de } \\
\text { dispersão }\end{array}$ & $\begin{array}{l}\text { Posição no } \\
\text { dossel }\end{array}$ & Ambiente \\
\hline \multirow[t]{3}{*}{ Sapindaceae } & Allophylus occidentalis (Sw.) Radlk. & & Nov./Dez. & & Anemocoria & dossel & mata manejada \\
\hline & Allophylus peruvianensis & Cabelo de cotia & Jan./Fev. & & Anemocoria & sub-bosque & mata nativa \\
\hline & Pseudima frutescens (Aubl.) Radlk. & Mata fome & Abr./Maio & Jul./Ago. & Zoocoria & sub-bosque & mata manejada \\
\hline \multirow[t]{3}{*}{ Sapindaceae } & Talisia mollis Kunt. ex Cambess. & Pitomba & Mar./Maio & Ago./Nov. & Zoocoria & dossel & $\begin{array}{l}\text { mata nativa e } \\
\text { manejada }\end{array}$ \\
\hline & Talisia retusa R.S. Cowan & $\begin{array}{l}\text { Pitomba de } \\
\text { macaco }\end{array}$ & & Out./Dez. & Zoocoria & dossel & $\begin{array}{l}\text { mata nativa e } \\
\text { manejada }\end{array}$ \\
\hline & Toulicia patentinervis Radlk. & Tipi & Jul./Ago. & & Anemocoria & dossel & mata manejada \\
\hline \multirow[t]{4}{*}{ Sapotaceae } & Pouteria híspida Eyma & $\begin{array}{l}\text { Tuturubá de } \\
\text { cancão }\end{array}$ & & Dez. & Zoocoria & dossel & mata manejada \\
\hline & Pouteria sagotiana (Baill.) Eyma & & Jun./Jul. & Jun./Ago. & Zoocoria & dossel & $\begin{array}{l}\text { mata nativa e } \\
\text { manejada }\end{array}$ \\
\hline & Pouteria sp.1 & Goiabão & Jun./Out. & Jul./Abr. & Zoocoria & dossel & $\begin{array}{l}\text { mata nativa e } \\
\text { manejada }\end{array}$ \\
\hline & Pouteria sp.2 & & & Ago./Set. & Zoocoria & dossel & mata manejada \\
\hline \multirow[t]{3}{*}{ Solanaceae } & Solanum asperum Vahl & Céga-jumento & Maio/Jul. & & Zoocoria & sub-bosque & mata manejada \\
\hline & Solanum caeruleum Vell. & & Jan./Abr. & & Zoocoria & sub-bosque & mata manejada \\
\hline & Solanum paludosum Moric. & Jurubebinha & Jul./Dez. & Out./Jul. & Zoocoria & ambos & $\begin{array}{l}\text { mata nativa e } \\
\text { manejada }\end{array}$ \\
\hline Theophrastaceae & Clavija latifolia K. Koch & Fruta de jaboti & Mar. & Mar./Jul. & Zoocoria & sub-bosque & mata nativa \\
\hline \multirow[t]{2}{*}{ Urticaceae } & Cecropia obtusa Trécul & $\begin{array}{l}\text { Imbaúba } \\
\text { branca }\end{array}$ & Abr. & & Zoocoria & dossel & mata manejada \\
\hline & Cecropia palmata Willd. & $\begin{array}{l}\text { Imbaúba } \\
\text { vermelha }\end{array}$ & Mar./Abr. & Mar./Maio & Zoocoria & dossel & mata manejada \\
\hline Violaceae & Rinorea ovalifolia (Britton) S.F. Blake & & Out./Dez. & Nov./Jan. & Zoocoria & ambos & $\begin{array}{l}\text { mata nativa e } \\
\text { manejada }\end{array}$ \\
\hline \multirow[t]{7}{*}{ Indeterminadas } & & Indet (1633) & Mar. & & Anemocoria & dossel & mata manejada \\
\hline & & Indet (10-441) & Nov./Dez. & & Zoocoria & sub-bosque & mata manejada \\
\hline & & Indet (253) & Mar. & & Anemocoria & dossel & mata manejada \\
\hline & & Indet (242) & Out./Dez. & & Zoocoria & dossel & mata nativa \\
\hline & & Indet (251) & Fev. & & Anemocoria & dossel & mata manejada \\
\hline & & Indet (256) & Abr. & & Anemocoria & dossel & mata manejada \\
\hline & & Indet (237) & & Out. & Anemocoria & dossel & mata nativa \\
\hline
\end{tabular}

Vários autores têm associado picos de floração em florestas amazônicas à estação seca (Araújo, 1970; Daunbemire, 1972; Alencar et al., 1979; Carvalho, 1980; Muniz, 1996; Haugaasen e Peres, 2005), embora existam dúvidas quanto aos fatores que controlam a periodicidade de floração das espécies que crescem nas regiôes de climas não sazonais (Alvim, 1964). Richards (1952) aponta a estação seca como o principal fator externo controlador da periodicidade rítmica da vegetação tropical. Araújo (1970) considera, ainda, que a floração e a frutificação ocorrem, na maioria dos casos, na dependência da distribuição das chuvas durante o ano, embora o efeito da regularidade ou severidade das estaçōes seca ou chuvosa sobre as plantas permaneça desconhecida (Newstrom et al., 1994).

As espécies do sub-bosque floresceram principalmente de fevereiro a julho, com máximo de floração em abril e maio, no fim da estação chuvosa (Fig. 2A), enquanto que as espécies do dossel floresceram principalmente de julho a dezembro, com máximo em agosto e outubro, no meio da estação seca, e as espécies de ambos os estratos floresceram de novembro a maio, com pico em dezembro, no início da estação chuvosa. Croat (1969) encontrou resultados semelhantes em floresta úmida no Panamá, com as espécies arbóreas do dossel exibindo o máximo da atividade de floração no fim da estação seca.

Quando se considera o número de indivíduos (Fig. 2B), observou-se um pico de floração em março (máximo da estação chuvosa) para as espécies do dossel e para as espécies de ambos os estratos, enquanto que as espécies do sub-bosque exibiram uma sazonalidade de floração distinta, com picos em fevereiro e em novembro. 
A frutificação nas espécies de sub-bosque não variou com a estação do ano, ao contrário das espécies de dossel e das espécies que ocorrem em ambos os estratos, as quais mostraram similaridade na sazonalidade da frutificação, com valores mais baixos no início da estação úmida (janeiro) e um aumento progressivo ao longo do ano, atingindo os maiores valores em julho (transição estação úmida/ estação seca), e na estação seca de outubro a dezembro (Fig. 3A).

A maioria dos indivíduos do sub-bosque frutificou durante a estação chuvosa, enquanto que os indivíduos do dossel frutificaram durante a estação seca (Fig. 3B).

Com 56 espécies (62,9\% do total), as espécies zoocóricas dominaram os padrōes de floração e frutificação nas duas áreas estudadas (Fig. 4). O período de maior floração ocorreu entre agosto e dezembro, correspondendo à estação seca e início da estação chuvosa, enquanto que o período de maior frutificação ocorreu de dezembro a julho, do início da estação chuvosa até a transição da estação chuvosa para a seca. Pesquisas têm sugerido que espécies zoocóricas apresentam um pico de frutificação durante a estação chuvosa, devido, talvez, a um aumento no nível de umidade necessário à produção dos frutos (Janzen, 1967; Lieberman, 1982; Chapman et al., 1999). As espécies anemocóricas (25, correspondendo a $28,1 \%$ do total) floresceram principalmente entre agosto
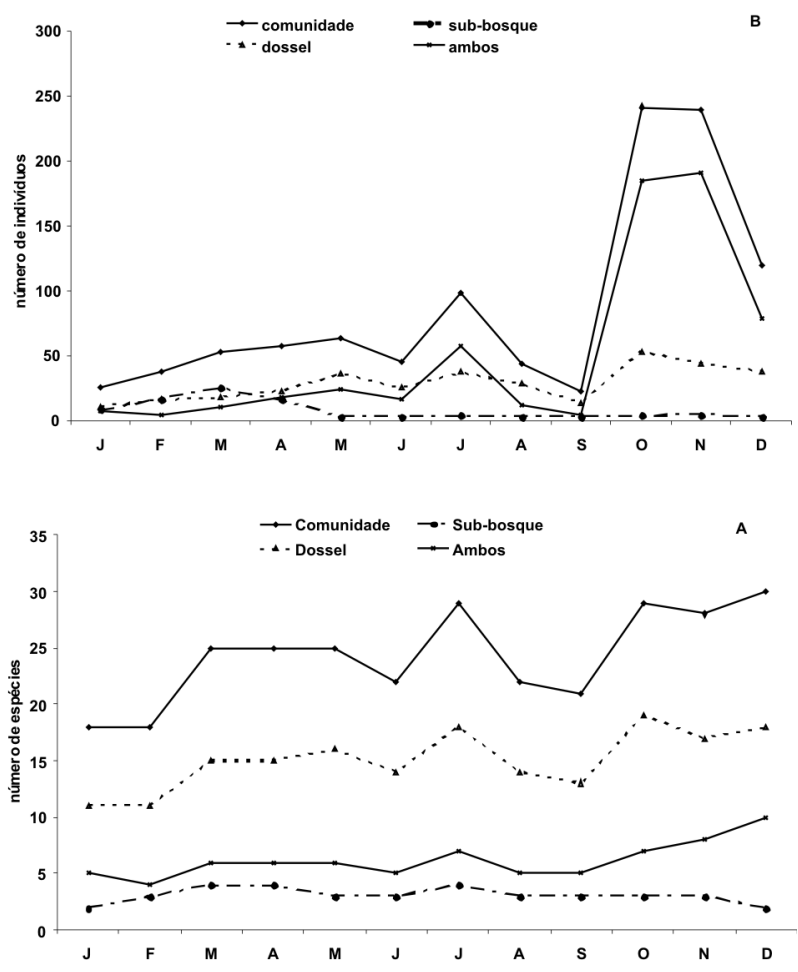

Figura 3 - Número de espécies arbóreas (A) e indivíduos (B) em frutificação da comunidade e por estrato (dossel, sub-bosque, e ambos (dossel e subbosque)) - Buriticupu, Maranhão, Sudoeste da Amazônia. e dezembro e frutificaram de setembro a dezembro, com máximo em outubro, que é o auge da estação seca. O número de indivíduos anemocóricos em frutificação (137) foi elevado no meio da estação seca.

Janzen (1967) e Costa (2003) afirmaram que, em florestas decíduas e semidecíduas, as espécies anemocóricas comumente maturam e liberam seus frutos no final da estação seca, quando os ventos são fortes e a perda de folhas é comum. Este é um comportamento bastante freqüente entre as árvores que alcançam o dossel e predomina entre as espécies emergentes, mas é raro entre plantas do sub-bosque.

As autocóricas, embora representem um percentual pequeno das espécies observadas (cerca de 9,0\%), floresceram de novembro a julho, do final da estação seca até a transição da estação chuvosa para a seca e frutificaram de julho a dezembro (estação seca, início da estação chuvosa).

Os picos de intensidade de floração e de frutificação ocorreram em março e outubro/novembro, respectivamente, tanto para as espécies zoocóricas como para as anemocóricas (Fig. 4).

Vários estudos têm estimado que mais de $80 \%$ das espécies de árvores e arbustos das florestas tropicais úmidas são dispersadas por animais (Frankie et al., 1974b; Croat, 1975; Hilty, 1980; Opler et al., 1980; Gentry, 1982). Saravy et al. (2003) também encontraram como maior ocorrência a zoocoria seguida pela anemocoria. Já as espécies dispersadas pelo vento são quase ausentes no sub-bosque (Opler $e t$ al., 1980), e também são raras entre as árvores (Gentry, 1982). Segundo Vieira et al. (2002), em florestas úmidas a propagação de sementes por anemocoria é baixa, por apresentar uma maior eficiência em áreas mais abertas. Em floresta ribeirinha, do total de espécies estudadas $72 \%$ apresentaram estratégia zoocórica, 24\% apresentaram estratégia anemocórica e 4\% eram autocóricas (Budke et al., 2005). Em floresta tropical, Kinoshita et al. (2006) também encontraram que a síndrome mais freqüente foi a zoocoria (63\%), seguida de anemocoria $(21 \%)$ e autocoria $(18 \%)$. Nesse mesmo trabalho, a zoocoria foi mais freqüente em árvores, arbustos e ervas, a maioria do sub-bosque; por outro lado, a anemocoria foi muito freqüente em lianas, e ocorreu em todos os estratos, com predomínio no estrato superior e a autocoria ocorreu em todos os hábitos e em todos os ambientes.

O número de espécies (Fig. 5A) e indivíduos (Fig. 5B) em floração e frutificação na mata manejada foi maior que na mata nativa em praticamente todos os meses do ano, mas tanto a floração quanto a frutificação demonstraram um ritmo sazonal mais definido na mata nativa que na manejada. De acordo com vários autores (Frankie et al., 1974b; Croat, 1975; Opler et al., 1980), a produção de frutos no dossel é mais sazonal que no sub-bosque. 

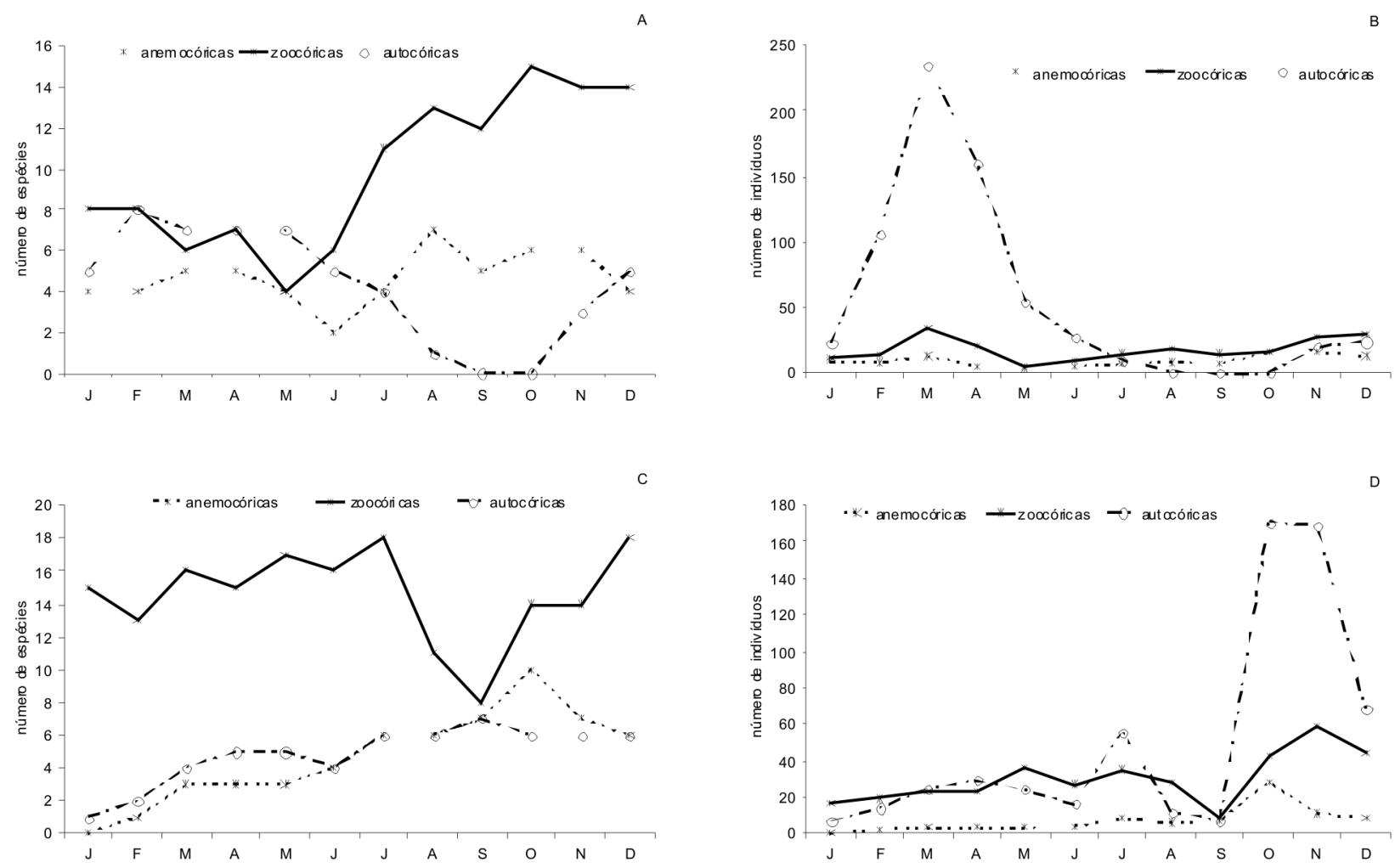

Figura 4 - Número de espécies arbóreas e indivíduos em floração (A e B) e frutificação (C e D), de acordo com o tipo de dispersão - Buriticupu, Maranhão, Sudoeste da Amazônia.

Aparentemente, o nível e intensidade da perturbação da floresta podem influenciar também os processos fenológicos, provavelmente relacionado à ocorrência de muitas espécies secundárias, de rápido crescimento na mata manejada. Entretanto, não foi observada diferença significativa entre as áreas (nativa e manejada), provavelmente devido à intensidade moderada da perturbação e ao tempo de recuperação da área (mais de dez anos).

A precipitação foi positivamente correlacionada à porcentagem de indivíduos em floração $(r=0,81 ; \mathrm{p}<0,001)$, espécies autocóricas em floração $(r=0,89 ; p<0,01)$, espécies do sub-bosque em floração $(r=0,57 ; p<0,05)$, espécies de ambos os estratos em floração $(r=0,68 ; p<0,02)$ e frutificação $(\mathrm{r}=0,92 ; \mathrm{p}<0,001)$. A precipitação foi negativamente correlacionada à porcentagem de espécies anemocóricas em frutificação $(r=0,86 ; \mathrm{p}<0,01)$, espécies zoocóricas em floração $(r=0,82 ; p<0,01)$, espécies autocóricas em frutificação $(r=0,78 ; p<0,01)$ e espécies do dossel em floração $(\mathrm{r}=0,63 ; \mathrm{p}<0,03)$.

Diferenças entre estação seca e chuvosa foram aparentes para a porcentagem de indivíduos em floração $(\mathrm{p}<0,001)$, espécies anemocóricas em floração e frutificação $(\mathrm{p}<0,01)$, indivíduos anemocóricos em frutificação $(\mathrm{p}<0,05)$, espécies zoocóricas em floração $(\mathrm{p}<0,05)$, espécies autocóricas em floração $(\mathrm{p}<0,01)$ e frutificação $(\mathrm{p}<0,05)$, espécies do dossel em floração $(p<0,01)$, espécies de ambos os estratos em floração $(\mathrm{p}<0,05)$ e indivíduos de ambos os estratos em floração $(\mathrm{p}<0,01)$.

O número médio de espécies anemocóricas florescendo e frutificando, espécies zoocóricas florescendo, espécies autocóricas frutificando e de espécies do dossel florescendo foi maior na estação seca, enquanto que o número médio de espécies zoocóricas frutificando, espécies autocóricas florescendo, e de espécies e indivíduos de ambos os estratos florescendo foi maior na estação chuvosa.

Os resultados obtidos no nível da comunidade demonstram uma grande sincronia na floração e frutificação dos indivíduos, e confirmam a relação entre esses processos e a variação na precipitação ao longo do ano, e também que plantas de dossel e sub-bosque exibem comportamentos fenológicos diferentes. As comparações entre as áreas (nativa e manejada) não demonstraram diferença significativa, provavelmente em função do tipo e intensidade da perturbação (corte seletivo) e ao tempo de recuperação da área (mais de dez anos).

\section{BIBLIOGRAFIA CITADA}



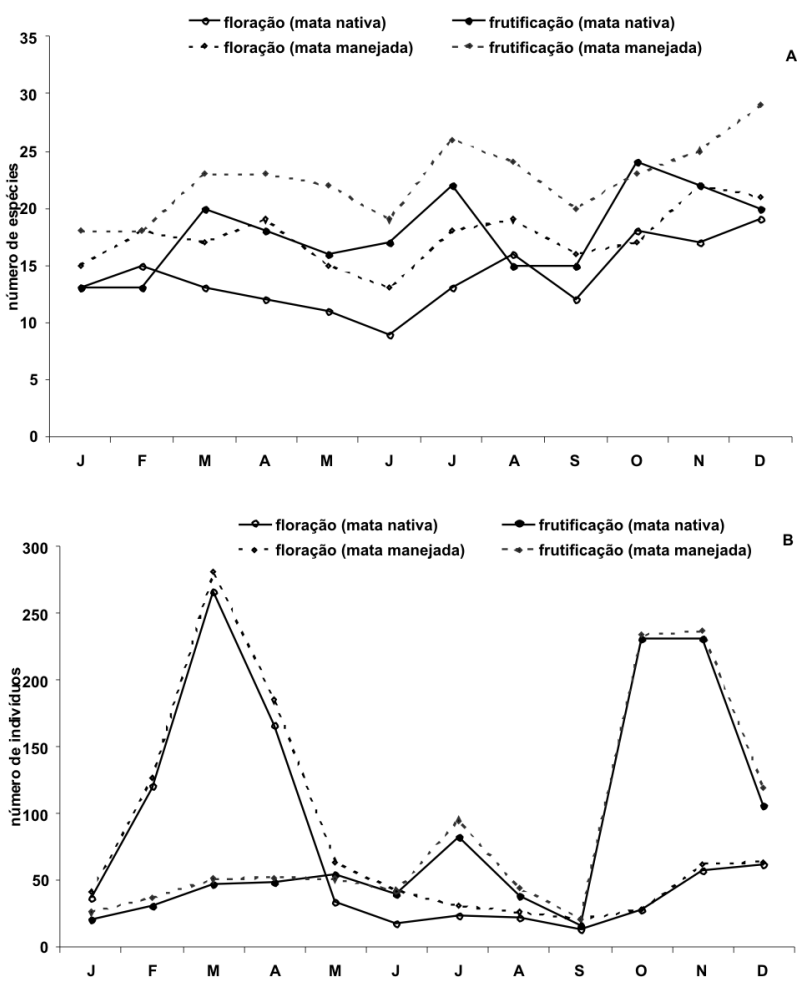

Figura 5 - Número de espécies arbóreas (A) e indivíduos (B) em floração e frutificação, na mata nativa e manejada - Buriticupu, Maranhão, Sudoeste da Amazônia.

Alencar, J. da C.; Almeida, R.A. de; Fernandes, N.P. 1979. Fenologia de espécies florestais em floresta tropical úmida de terra firme na Amazônia Central. Acta Amazonica, 9(1):163-198.

Alvim, P. de T. 1964. Periodicidade do crescimento das árvores em climas tropicais. Anais do XI Congresso Nacional de Botânica, 405-422.

Araújo, V.C. de. 1970. Fenologia de essências florestais amazônicas I. INPA, Manaus, 25p. (Boletim do INPA, 4).

Ashton, P.S.; Givinish, T.J.; Appanah, S. 1988. Staggered flowering in the Dipterocarpaceae: new insights into floral induction and the evolution of mast fruiting in the aseasonal tropics. American Naturalist, 132:44-66.

Brasil. 1986. Levantamento exploratório - Reconhecimento de solos do Estado do Maranhão. vol. 1. EMBRAPA/SNLCS: Rio de Janeiro (Boletim de Pesquisa, 35). 522p.

Bencke, C.S.C.; Morellato, L.P.C. 2002. Comparação de dois métodos de avaliação de plantas, sua interpretação e representação. Revista Brasileira de Botânica, 25(3):269-275.

Budke, J.C.; Athayde, E.A.; Giehl, E.L.H.; Záchia, R.A.; Eisinger, S.M. 2005. Composição florística e estratégias de dispersão de espécies lenhosas em uma floresta ribeirinha, arroio Passo das Tropas, Santa Maria, RS, Brasil. Iheringia, Sér. Bot., 60(1):1724.
Carvalho, J.O.P. de. 1980. Fenologia de espécies florestais de potencial econômico que ocorrem na Floresta Nacional do Tapajós. EMBRAPA/CPATU, Belém, 15p. (Boletim de Pesquisa, 20).

Chapman, C.A.; Wrangham, R.W.; Chapman, L.J.; Kennard, D.K.; Zanne, A.E. 1999. Fruit and flower phenology at two sites in Kibale National Park, Uganda. Journal of Tropical Ecology, 15:189-211.

Chapman, C.A.; Chapman, L.J.; Struhsaker, T.T.; Zanne, A.E.; Clark, C.J.; Poulsen, J.R. 2005. A long-term evaluation of fruiting phenology: importance of climate change. Journal of Tropical Ecology, 21:31-45.

Charles-Dominique, P.; Atramentowicz, M.; Charles-Dominique, M.; Gerard, H.; Hladik, A.; Hladik, C.M.; Prevost, M.F. 1981. Les mammiferes frugivores arboridoles nocturne d'une forest guyanaise: interrelations plantes-animaux. Revue d'Ecologie (Terre et Vie, 35:341- 436

Costa, F. A. P.L. 2003. Ecologia, evolução e o valor das pequenas coisas. Edição do Autor, Juiz de Fora, 137p.

Croat, T.B. 1969. Seasonal flowering behavior in Central Panama. Ann. Miss. Bot. Garden, 56:295-307.

Croat, T.B. 1975. Phenological behavior of habitat and habitat classes on Barro Colorado Island (Panama Canal Zone). Biotropica, $7(4): 270-277$

Daubenmire, B. 1972. Phenology and others characteristics of tropical semideciduous forest in North-Western Costa Rica. J. Ecol., 60:147-170.

Fischer, K.E.; Chapman, C.A. 1993. Frugivores and fruit syndromes: differences in patterns at the genus and species level. Oikos, 66:472-482.

Foster, R.B. 1985. The seasonal rhythm of fruitfall on Barro Colorado Island. In: Lieth Jr, E.G., Rand, A.S.; Windsor, D.M. (eds.). The ecology of a tropical forest: seasonal rhythms and long-term changes. Smithsonian Institution Press: Washington, pp.151-172.

Frankie, G.W.; Baker, H.G.; Opler, P.A. 1974a. Comparative phenological studies of trees in tropical wet and dry forest in the lowlands of Costa Rica. J. Ecol., 62:881-919.

Frankie, G.W.; Baker, H.G.; Opler, P.A. 1974b. Tropical plant phenology: applications for studies in community ecology. In: Lieth, H. (ed.). Phenology and seasonality modeling. SpringerVerlag, New York. p.287-296.

Gentry, A.H. 1982. Patterns of Neotropical plant species diversity. Evol. Biol., 15:1-84.

Golfari, L. 1980. Zoneamento ecológico para reflorestamento da área de influência da Serra de Carajás. Revista da CVRD, 1(2):8-18.

Gribel, R.; Gibbs, P.E.; Queiróz, A.L. 1999. Flowering phenology and pollination biology of Ceiba pentandra (Bombacaceae) in Central Amazonia. Journal of Tropical Ecology, 15:247-263.

Haugaasen, T.; Peres, C.A. 2005. Tree phenology in adjacent Amazonian flooded and unflooded forests. Biotropica, 37(4): 620-630.

Hilty, S.L. 1980. Flowering and fruiting periodicity in a pre-montane rain forest in Pacific Colombia. Biotropica, 12:292-306. 
Janzen, D.H. 1967. Synchronization of sexual reproduction of trees within the dry season in Central America. Evolution, 21: 620-637.

Jesus, R.M. de; Menandro, M.S.; Thibau, C.E. 1986. Manejo florestal em Buriticupu. Anais 1 Simpósio do Trópico Úmido. EMBRAPA/CPATU: Belém. p.245-251.

Kinoshita, L.S.; Torres; R.B.; Forni-Martins, E.R.; Spinelli, T.; Ahn, Y.J.; Constâncio, S.S. 2006. Composição florística e síndromes de polinização e de dispersão da mata do Sítio São Francisco, Campinas, SP, Brasil. Acta Botanica Brasilica, 20(2):313-327.

Lieberman, D. 1982. Seasonality and phenology in a dry tropical forest in Ghana. Journal of Ecology, 70:791-806.

Longman, K.A.; Jenik, J. 1987. Tropical forest and its environment. Longman Scientific and Technical, Singapore. 358p.

Macêdo, M. 1977. Dispersão de plantas lenhosas de uma campina amazônica. Acta Amazonica, 7(1): 1-69. (Suplemento).

Muniz, F.H. 1996. Floração e frutificação das árvores da Reserva Florestal do Sacavém em São Luís - MA. Pesquisa em Foco, $4(4): 46-61$.

Muniz, F.H. 1998. Estrutura e dinâmica da floresta pré-amazônica na Reserva Florestal de Buriticupu, Buriticupu - MA. Tese de Doutorado, Instituto de Biociências/UNESP, Rio Claro, 228p.

Newbery, D.M.; Songwe, N.C.; Chuyong, G.B. 1998. Phenology and dynamics of an African rainforest at Korup, Cameroon. In: Newbery, D.M.; Prins, H.H.T.; Brown, N.D. (eds). Dynamics of tropical communities. Blackwell Science, Oxford. p. 267-308.

Newstron, L.E.; Frankie, G.W.; Baker, H.G.; Colwell, R.K. 1994. Diversity of long-term flowering patterns. In: McDade, L.A.; Bawa, K.S.; Hespenheide, H.A.; Hartshirn, G.S. (eds.), La Selva: ecology and natural history of a neotropical rain forest. Chicago University Press, Chicago. p.142-160.

Opler, P.A.; Frankie, G.W.; Baker, H.G. 1976. Rainfall as a factor in the release, timing, and synchronization of anthesis by tropical trees and shrubs. Journal of Biogeography, 3:231-236.

Opler, P.A.; Frankie, G.W.; Baker, H.G. 1980. Comparative phenological studies of treelet and shrub species in tropical wet and dry forest in the lowlands of Costa Rica. Journal of Ecology, 68:167-188.

Pijl, L. van der. 1982. Principles of dispersal in higher plants. $3^{\text {th }} \mathrm{ed}$. Springer-Verlag., New York. 215p.

Pinto, A.M.; Ribeiro, R.A.; Alencar, J. da C.; Barbosa, A.P. 2005. Fenologia de Simarouba amara Aubl. na Reserva Florestal Adolpho Ducke, Manaus, AM. Acta Amazonica, 35(3):347352.

Ramos, D.R.; Soares, C.S.; Ferreira, C.M.; Santos, M.D. 1991. Caracterização dos solos do corredor da Estrada de Ferro Carajás, sob diferentes formas de ocupação. UFRRJ/DS: Publicação Especial. 87p.

Rathke, B.; Lacey, E.P. 1985. Phenological patterns of terrestrial plants. Annual Review of Ecology and Systematics, 16:179-214.
Richards, P.W. 1952. The tropical rain forest. Cambridge University Press, Cambridge. 423p.

Roosmalen, M.G.M. van. 1985. Fruits of Guianan Flora. Institute of Systematic Botany, Utrecht University: Utrecht. 483p.

Saravy, F.P.; Freitas, P.J. de; Lage, M.A.; Leite, S.J.; Braga, L.F.; Sousa, M.P. 2003. Síndrome de dispersão em estratos arbóreos em um fragmento de floresta ombrófila aberta e densa em Alta Floresta - MT. Revista do Programa de Ciências Agro-Ambientais, Alta Floresta, 2(1):1-12.

Sarmiento, G.; Monastério, M. 1983. Life forms and phenology. In: Bourliere, F. (ed.). Tropical savannas. Elsevier Scientific Publishing Company, Amsterdam. p.79-108.

Smythe, N. 1970. Relationships between fruiting seasons and seed dispersal methods in a neotropical forest. American Naturalist, 104:25-35.

Snow, D.W. 1965. A possible selective factor in the evolution of fruiting seasons in tropical forest. Oikos, 15:274-281.

Sun, C.; Kaplin, B.A.; Kristensen, K.A.; Munyaligoga, V.; Mvukiyumwami, J.; Kajondo, K.K.; Moermond, T.C. 1996. Tree phenology in a tropical montane forest in Rwanda. Biotropica, 28:668-681.

Tutin, C.E.G.; Fernandez, M. 1993. Relationships between minimum temperature and fruit production in some tropical forest trees in Gabon. Journal of Tropical Ecology, 9:241-248.

Van Schaik, C.P. 1986. Phenological changes in a Sumatran rain forest. Journal of Tropical Ecology, 2:327-347.

Van Schaik, C.P.; Terborgh, J.W.; Wright, S.J. 1993. The phenology of tropical forests: adaptive significance and consequences for primary consumers. Annual Review of Ecology and Systematics, 24:353-377.

Vieira, D.L.M.; Aquino, F.G.; Brito, M.A.; Bulhão, C.F.; Henriques, R.P.B. 2002. Síndrome de dispersão de espécies aburstivoarbóreas em cerrado sensu strictu do Brasil Central e savanas amazônicas. Revista Brasileira de Botânica, 25(2):215-220.

Walter, H. 1986. Vegetação e zonas climáticas: tratado de ecologia global. EPU, São Paulo. 108p.

Wheelwright, N.T. 1985. Competition for dispersers, and the timing of flowering and fruiting in a guild of tropical trees. Oikos, 44:465-477.

Whitmore, T.C. 1984. Tropical rain forests of the far east. 2nd ed. Claredon Press, Oxford. 352p.

Zar, J.H. 1996. Biostatistical analysis. 3rd. edition. Prentice-Hall International Editions, New Jersey. 662p.

Zimmerman, J.K.; Wright, S.J.; Calderón, O.; Pagan, M.A.; Paton, S. 2007. Flowering and fruiting phenologies of seasonal and aseasonal neotropical forests: the role of annual changes in irradiance. Journal of Tropical Ecology, 23:231-251.

Recebido em 08/09/2005

Aceito em 21/10/2008 